\title{
Two Transiting Hot Jupiters from the WASP Survey: WASP-150b and WASP-176b
}

\author{
Benjamin F. Cooke ${ }^{1,2}$ (1) , Don Pollacco ${ }^{1,2}$ (1) , Y. Almleaky ${ }^{3,4}$, K. Barkaoui ${ }^{5,6}$, Z. Benkhaldoun ${ }^{6}$, James A. Blake ${ }^{1,2}$,
} François Bouchy $^{7}$, Panos Boumis ${ }^{8}$, D. J. A. Brown ${ }^{1,2}$ (1) Ivan Bruni $^{9}$, A. Burdanov ${ }^{5}$, Andrew Collier Cameron ${ }^{10}$ (1), Paul Chote ${ }^{1,2}$, A. Daassou ${ }^{6}$, Giuseppe D'ago ${ }^{11}$ (1), Shweta Dalal ${ }^{12}$, Mario Damasso ${ }^{13}$, L. Delrez ${ }^{14}$, A. P. Doyle ${ }^{1}$, E. Ducrot ${ }^{5}$, M. Gillon ${ }^{5}$, G. Hébrard ${ }^{12}$, C. Hellier ${ }^{13}$ (1) Thomas Henning $^{15}$, E. Jehin ${ }^{16}$, Flavien Kiefer ${ }^{12}$, George W. King ${ }^{1,2}$, Alexios Liakos ${ }^{8}$ (1), Théo Lopez $^{17}$, Luigi Mancini ${ }^{13,15,18,19}$ (10), Rosemary Mardling ${ }^{7,20}$, P. F. L. Maxted ${ }^{21}$ (1) , James McCormac $^{1,2}$, C. Murray ${ }^{14}$, Louise D. Nielsen ${ }^{7}$, Hugh Osborn ${ }^{17,22}$, E. Palle ${ }^{23,24}$, Francesco Pepe ${ }^{7}$, F. J. Pozuelos ${ }^{5,16}$ (1), J. Prieto-Arranz ${ }^{23,24}$, D. Queloz ${ }^{7,14}$ (1), Nicole Schanche ${ }^{10}$ (1) , Damien Ségransan ${ }^{7}$, Barry Smalley ${ }^{21}$ (10), John Southworth ${ }^{21}$, S. Thompson ${ }^{14}$, Oliver Turner ${ }^{7}$, Stéphane Udry ${ }^{7}$ (10), S. Velasco ${ }^{23,24}$, Richard West ${ }^{1,2}$, Pete Wheatley ${ }^{1,2}$ (i), and John Alikakos ${ }^{8}$

${ }^{1}$ Department of Physics, University of Warwick, Gibbet Hill Road, Coventry CV4 7AL, UK; b.cooke@ warwick.ac.uk

${ }_{2}$ Centre for Exoplanets and Habitability, University of Warwick, Gibbet Hill Road, Coventry CV4 7AL, UK

${ }^{3}$ Space and Astronomy Department, Faculty of Science, King Abdulaziz University, 21589 Jeddah, Saudi Arabia

${ }^{4}$ King Abdullah Centre for Crescent Observations and Astronomy, Makkah Clock, Mecca 24231, Saudi Arabia ${ }^{5}$ Astrobiology Research Unit, Université de Liège, Belgium

${ }^{6}$ Oukaimeden Observatory, High Energy Physics and Astrophysics Laboratory, Cadi Ayyad University, Marrakech, Morocco

${ }^{7}$ Observatoire astronomique de l'Université de Geneve, $51 \mathrm{ch}$. des Maillettes, 1290 Sauverny, Switzerland

${ }^{8}$ Institute for Astronomy, Astrophysics, Space Applications and Remote Sensing, National Observatory of Athens, 15236 Penteli, Greece 9 INAF-Osservatorio Astronomico di Bologna, Via Ranzani 1, I-40127 Bologna, Italy

${ }^{10}$ Centre for Exoplanet Science, SUPA, School of Physics and Astronomy, University of St. Andrews, North Haugh, St. Andrews KY16 9SS, UK

${ }^{11}$ Instituto de Astrofísica, Facultad de Física, Pontificia Universidad Católica de Chile, Av. Vicuña Mackenna 4860, 7820436 Macul, Santiago, Chile

${ }^{12}$ Institut d'Astrophysique de Paris, UMR7095 CNRS, Universite Pierre \& Marie Curie, 98bis boulevard Arago, F-75014 Paris, France

${ }^{13}$ INAF-Osservatorio Astrofisico di Torino, via Osservatorio 20, I-10025, Pino Torinese, Italy

${ }^{14}$ Cavendish Laboratory, J J Thomson Avenue, Cambridge CB3 OHE, UK

${ }^{15}$ Max Planck Institute for Astronomy, Königstuhl 17, D-69117, Heidelberg, Germany

${ }^{16}$ Space sciences, Technologies and Astrophysics Research (STAR) Institute, Université de Liège, Belgium

${ }^{17}$ Aix Marseille Univ, CNRS, CNES, LAM, Marseille, France

${ }^{18}$ Department of Physics, University of Rome Tor Vergata, Via della Ricerca Scientifica 1, I-00133, Rome, Italy

${ }^{19}$ International Institute for Advanced Scientific Studies (IIASS), Via G. Pellegrino 19, I-84019, Vietri sul Mare (SA), Italy

${ }^{20}$ School of Physics and Astronomy, Monash University, Victoria, 3800, Australia

${ }^{21}$ Astrophysics Group, Lennard-Jones Laboratories, Keele University, Staffordshire ST5 5BG, UK

${ }^{22}$ Center for Space and Habitability, University of Bern, Gesellschaftsstrasse 6, 3012 Bern, Switzerland

23 Space and Habitability, University of Bern, Gesellschaftsstrasse 6, 3012 Bern, Swit
Instituto de Astrofísica de Canarias (IAC), E-38205 La Laguna, Tenerife, Spain

${ }^{24}$ Departamento de Astrofísica, Universidad de La Laguna (ULL), E-38206 La Laguna, Tenerife, Spain

Received 2020 January 21; revised 2020 April 9; accepted 2020 April 11; published 2020 May 7

\begin{abstract}
We report the discovery of two transiting exoplanets from the WASP survey, WASP-150b and WASP-176b. WASP$150 \mathrm{~b}$ is an eccentric $(e=0.38)$ hot Jupiter on a 5.6 day orbit around a $V=12.03$, F8 main-sequence host. The host star has a mass and radius of $1.4 M_{\odot}$ and $1.7 R_{\odot}$ respectively. WASP-150b has a mass and radius of $8.5 M_{\mathrm{J}}$ and $1.1 R_{\mathrm{J}}$, leading to a large planetary bulk density of $6.4 \rho_{\mathrm{J}}$. WASP- $150 \mathrm{~b}$ is found to be $\sim 3 \mathrm{Gyr}$ old, well below its circularization timescale, supporting the eccentric nature of the planet. WASP-176b is a hot Jupiter planet on a 3.9 day orbit around a $V=12.01, \mathrm{~F} 9$ sub-giant host. The host star has a mass and radius of $1.3 M_{\odot}$ and $1.9 R_{\odot}$. WASP-176b has a mass and radius of $0.86 M_{\mathrm{J}}$ and $1.5 R_{\mathrm{J}}$, respectively, leading to a planetary bulk density of $0.23 \rho_{\mathrm{J}}$.
\end{abstract}

Unified Astronomy Thesaurus concepts: Exoplanet systems (484); Radial velocity (1332); Exoplanet detection methods (489); Photometry (1234)

Supporting material: data behind figures

\section{Introduction}

As of 2019 October, over 4000 exoplanets have been verified. $^{25}$ Of these planets, over 3000 have been discovered using the transit method. These results have shown that exoplanet populations are both very common and very diverse (Batalha 2014), with a wide range of system parameters found thus far. The transit discoveries have been made using both spacebased surveys, for example Kepler/K2 (Borucki et al. 2010; Howell et al. 2014), and ground-based surveys, including the Wide Angle Search for Planets (WASP; Pollacco et al. 2006),

\footnotetext{
${ }^{25}$ https://exoplanetarchive.ipac.caltech.edu/
}

HATNet/HATSouth (Bakos 2018), Kilodegree Extremely Little Telescope (KELT; Pepper et al. 2007), TRAnsiting Planets and PlanetesImals Small Telescope (TRAPPIST; Jehin et al. 2011), and Next-Generation Transit Survey (NGTS; Wheatley et al. 2018). This field is expected to be expanded upon even further in the coming years with the yield of the Transiting Exoplanet Survey Satellite (TESS; Ricker et al. 2015) and other future planned missions such as the James Webb Space Telescope (Gardner et al. 2006), Planetary Transits and Oscillations of stars (PLATO; Rauer et al. 2016), and Atmospheric Remote-sensing Infrared Exoplanet Large-survey (ARIEL; Pascale et al. 2018).

Within this sample of exoplanets lies the region corresponding to hot Jupiters. A type of planet noticeably absent from 
our own solar system, hot Jupiters are planets with masses comparable to Jupiter but with orbital periods on the order of days. Current estimates put the occurrence rate of giant planets within 5-10 au around FGK stars at 10\%-20\% (Cumming et al. 2008; Mayor et al. 2011). Further detections and precise characterizations of hot Jupiter exoplanets will improve our ability to study this population using statistical methods, and hence allow for a better understanding of this unique type of planet.

Exoplanets are broadly characterized using a combination of the transit method (Charbonneau et al. 2000; Henry et al. 2000; Winn 2010) and radial velocity (RV) measurements. When combined these methods allow for the measurement of radius, mass, and density, which can lead to inferences of composition.

This paper discusses the discovery and characterization of the transiting hot Jupiters WASP-150b and WASP-176b. Sections 2-4 detail the WASP discovery, spectroscopic follow-up, and photometric follow-up, respectively. Section 5 discusses the high spatial resolution follow-up of WASP-150. Section 6 outlines the analysis and derived parameters. Finally, Section 7 summarizes the discovery findings.

\section{SuperWASP Discovery Photometry}

The WASP project (now decommissioned) was split into north and south facilities with telescopes located at the Isaac Newton Group at the Observatorio del Roque de los Muchachos, La Palma, Spain and at the South African Astronomical Observatory, Sutherland, Republic of South Africa respectively. Both facilities consisted of 8 Canon $200 \mathrm{~mm} f / 1.8$ lenses, each linked to an Andor e2v $2048 \times 2048$ pixel CCD. Each camera had a total field of view of $7.8 \times 7^{\circ} .8$ with a pixel scale of 13 !! 7 (Pollacco et al. 2006).

For WASP-150, a total of 99,892 photometric data points were taken between 2004 May 14 and 2011 August 4. These data ranged across 38 transits. WASP-150b was flagged as a high priority candidate on 2014 February 17 and confirmed as a planet on 2015 June 25. Figure 1 shows the WASP discovery curve folded by the best-fit period and binned to 10 minutes. Additionally we show the best-fit transit model from our Markov Chain Monte Carlo (MCMC).

For WASP-176, a total of 23,082 photometric data points were taken between 2004 May 26 and 2010 October 6. These data ranged across 30 transits. WASP-176b was flagged as a high priority candidate on 2014 February 12 and confirmed as a planet on 2018 January 23. Figure 2 shows the WASP discovery curve folded by the best-fit period and binned to 10 minutes. Additionally we show the best-fit transit model from our MCMC.

The SuperWASP data were reduced using the standard SuperWASP pipeline as described in Pollacco et al. (2006). Analysis of the light curve was then carried out using the box least-squares (BLS) fit method, as in Kovács et al. (2002), and the SysRem detrending algorithm, described in Tamuz et al. (2005). The results of BLS searches on the detrended data are shown in Figures 1 and 2. The data were searched with a transit-search algorithm (Collier Cameron et al. 2007b) and flagged as belonging to a planetary candidate. System parameters were then estimated from catalog data and a Monte Carlo simulation (Collier Cameron et al. 2006). These initial estimates produced a period of 5.644 days, a depth of
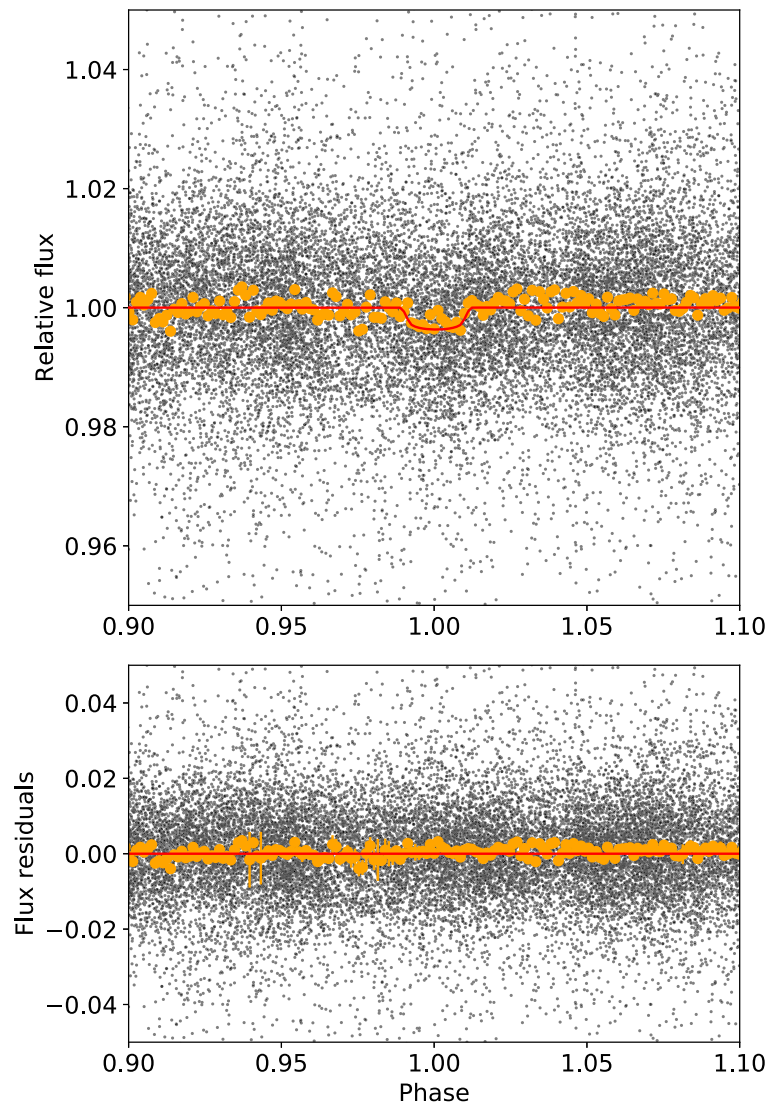

(a) Folded photometry

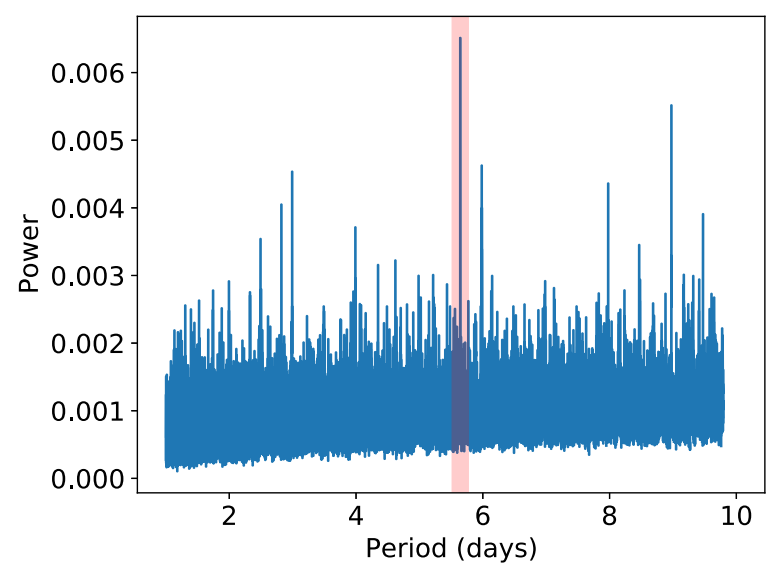

(b) BLS periodogram

Figure 1. (a) Upper panel: phase-folded WASP data for WASP-150b binned to 10 minutes. Data are shown in orange with the best-fit model derived from the MCMC analysis shown in red. Lower panel: residuals from the MCMC fit. (b) BLS periodogram of the WASP photometry. The period from our MCMC analysis is highlighted.

(The data used to create this figure are available.)

$3.2 \mathrm{mmag}$, and a width of $2.4106 \mathrm{hr}$ for WASP-150b and a period of 3.899 days, a depth of $3.4 \mathrm{mmag}$, and a width of $4.5292 \mathrm{hr}$ for WASP-176b. Additional follow-up spectroscopy and photometry were then obtained to confirm and characterize the planets. Basic photometric properties of the two hosts are shown in Table 1. 

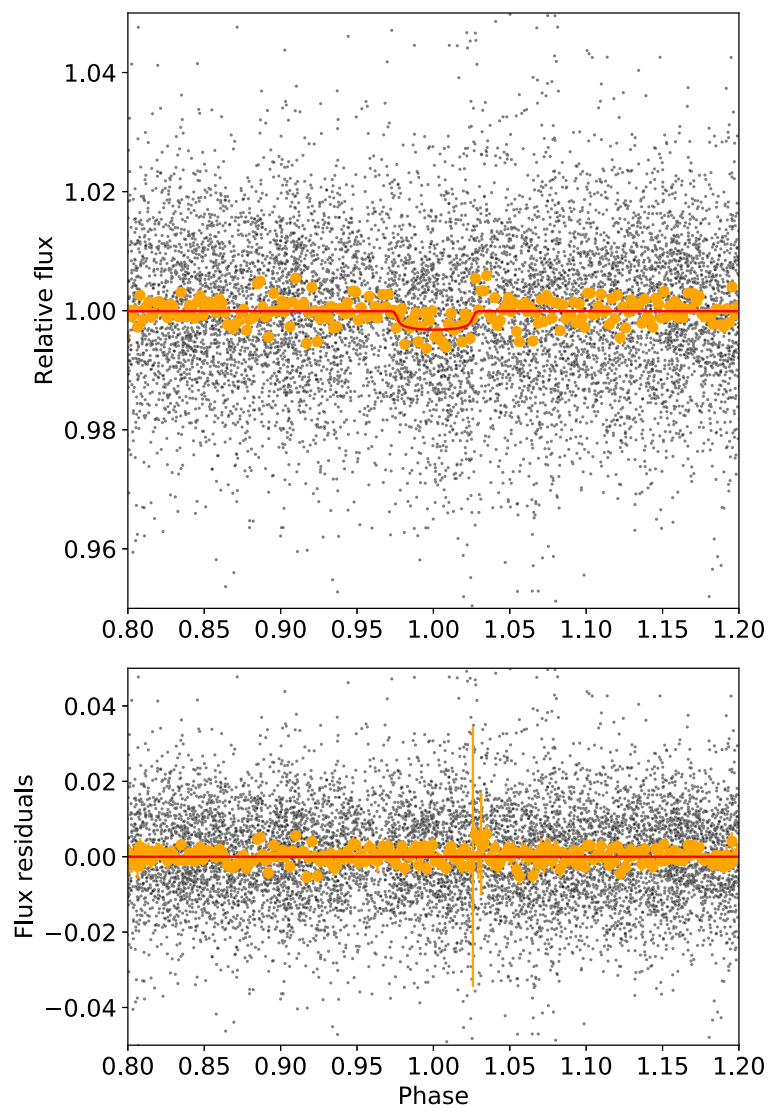

(a) Folded photometry

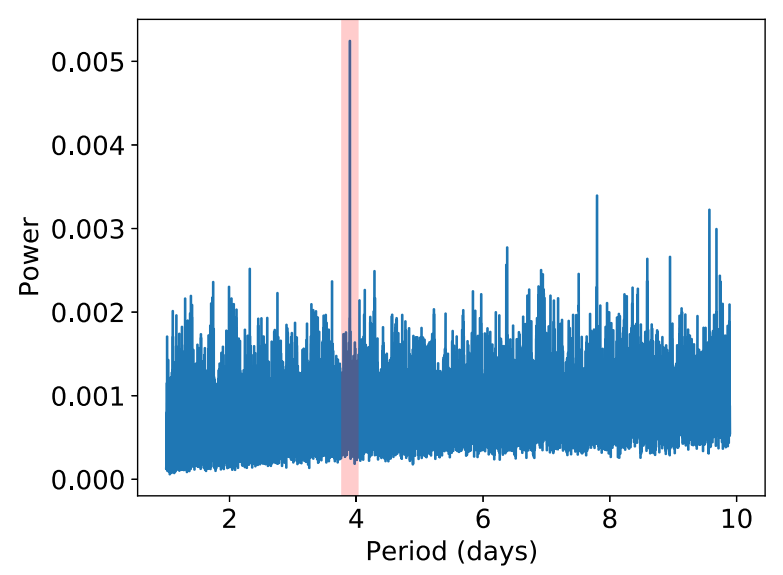

(b) BLS periodogram

Figure 2. (a) Upper panel: phase-folded WASP data for WASP-176b binned to 10 minutes. Data are shown in orange with the best-fit model derived from the MCMC analysis shown in red. Lower panel: residuals from the MCMC fit. (b) BLS periodogram of the WASP photometry. The period from our MCMC analysis is highlighted.

(The data used to create this figure are available.)

\section{Spectroscopic Follow-up}

\subsection{WASP-150b}

WASP-150 was observed with the SOPHIE spectrograph, first to establish the planetary nature of the transiting candidate, then to characterize the secured planet by measuring in particular its mass and orbital eccentricity. SOPHIE is dedicated to high-
Table 1

Photometric Properties

\begin{tabular}{lcc}
\hline \hline Parameter & WASP-150 & WASP-176 \\
\hline R.A. & $17: 37: 03.14$ & $20: 54: 44.94$ \\
Decl. & $+53: 01: 16.4$ & $+09: 10: 44.5$ \\
$V$ & 12.03 & 12.01 \\
$J$ & 11.06 & 10.99 \\
$G$ & 11.92 & 11.94 \\
\hline
\end{tabular}

precision RV measurements at the $1.93 \mathrm{~m}$ telescope of the Haute-Provence Observatory (Perruchot et al. 2008; Bouchy et al. 2009) and is widely used for WASP follow-up (e.g., Collier Cameron et al. 2007a; Hébrard et al. 2013; Schanche et al. 2019). We used its high-efficiency mode with a resolving power of $R=40,000$ and slow readout mode. We obtained 22 observations between 2014 May and 2015 April. Depending on weather conditions, exposure times ranged between 8 and 33 minutes in order to maintain a signal-to-noise ratio as constant as possible among observations.

The spectra were extracted using the SOPHIE pipeline (Bouchy et al. 2009), and the RVs were measured through weighted cross-correlation with a numerical mask (Baranne et al. 1996; Pepe et al. 2002). They were corrected for the CCD charge transfer inefficiency (Bouchy et al. 2009), and their error bars were computed from the cross-correlation function (CCF) using the method presented by Boisse et al. (2010). The monitoring of constant stars revealed no significant instrumental drifts during the epochs of observation, and none of the spectra were significantly affected by any moonlight or other sky background pollution.

The resulting CCFs have FWHM of $14.1 \pm 0.1 \mathrm{~km} \mathrm{~s}^{-1}$, and contrast that represents $\sim 14 \%$ of the continuum. The lines are slightly broader than what is usually measured in the highefficiency mode due to the stellar rotation of WASP-150 (we measured a projected rotational velocity $v \sin i_{*}=8.3 \pm 1.0$ $\mathrm{km} \mathrm{s}^{-1}$ from the parameters of the CCF using the calibration of Boisse et al. 2010).

The RVs have uncertainties around $19 \mathrm{~m} \mathrm{~s}^{-1}$. They show large variations in phase with the SuperWASP transit ephemeris for an eccentric orbit, and a semi-amplitude of the order of $800 \mathrm{~m} \mathrm{~s}^{-1}$. This would correspond to a companion in the massive-planet regime. The SOPHIE RVs are shown in Figure 3 with the best-fit model and residuals from our MCMC analysis (see Section 6.1.2). Data files can be found at https:// github.com/BenCooke95/W150-W176.

Radial velocities measured using different stellar masks (F0, $\mathrm{G} 2, \mathrm{~K} 0$, or K5) produce variations with similar amplitudes, so it is unlikely that these variations are produced by blend scenarios composed of stars of different spectral types. Similarly, the measured CCF bisector spans quantify possible shape variations of the spectral lines. They show a low dispersion of $27 \mathrm{~m} \mathrm{~s}^{-1}$, which agrees with their expected accuracy and is tiny in comparison to the large RV variations. We can thus conclude that the RV variations are not due to spectral-line profile changes attributable to blends or stellar activity, but rather to Doppler shifts due to a massive, eccentric planetary companion. Figure 4 shows the bisector spans.

\subsection{WASP-176b}

High resolution spectroscopy for WASP-176 was performed with the CORALIE spectrograph (Queloz et al. 2000; 

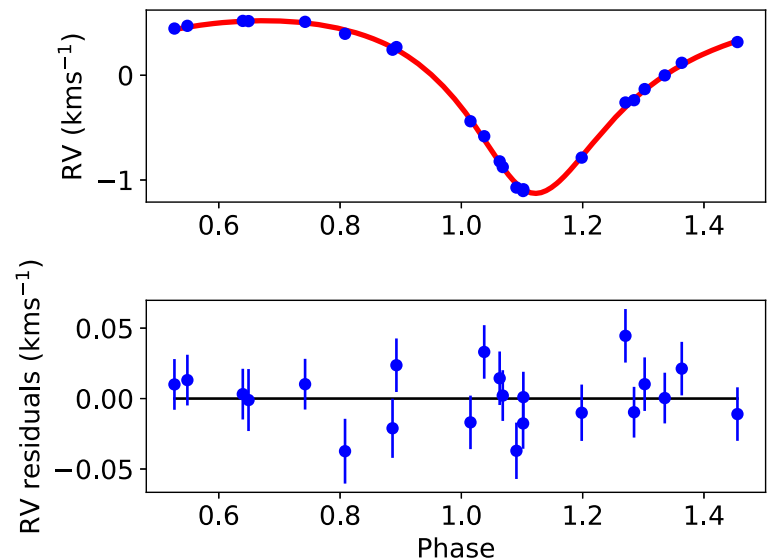

Figure 3. Upper panel: phase-folded SOPHIE RV observations of WASP-150. Data are shown in blue with the best-fit model derived from the MCMC analysis shown in red. Lower panel: residuals from the RV fit.

(The data used to create this figure are available.)

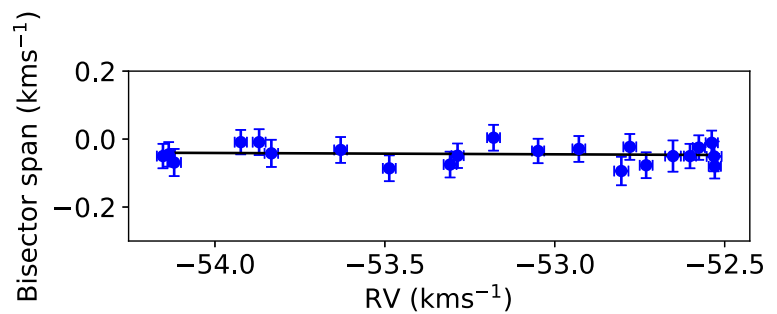

Figure 4. SOPHIE RV bisector span as a function of RV for WASP-150. The solid black line shows the best weighted linear fit to the data. The lack of any significant gradient supports the assumption that the RV signal is produced by a planetary companion. The aspect ratio of the two axes is unity.

Pepe et al. 2017) on the Swiss $1.2 \mathrm{~m}$ telescope at La Silla Observatory (Chile). In total we obtained 26 measurements between 2014 and 2018 July. RVs were computed with the standard CORALIE data reduction pipeline by cross-correlating the spectra with a binary G2 mask (Pepe et al. 2002).

The reduced CORALIE data are displayed in Figure 5 along with the best-fit model and residuals from our MCMC analysis (see Section 6.2.2). Data files can be found at https://github. com/BenCooke95/W150-W176.

Additionally, the line bisector was analyzed to ensure that the observed signal was indeed from an orbiting body and not a blended binary (Queloz et al. 2001). Figure 6 shows the results of this analysis using the bisector velocity span as a function of $\mathrm{RV}$. No significant correlation is seen (evidenced by the best-fit linear slope). Thus, this analysis supports the detection of a planetary companion to WASP-176. Using the Grubbs test for outliers (Grubbs 1950) we found exactly one outlier at $95 \%$. This point was removed before calculating the line bisector correlation.

\section{Photometric Follow-up}

\subsection{WASP- $150 b$}

A number of telescopes were used to gather the necessary follow-up photometry for WASP-150b. These are summarized in Table 2 along with observation dates, photometric filters, and transit notes. The following sections detail the follow-up. The light curves are shown in Figures 7 and 8 . Data files can be found at https://github.com/BenCooke95/W150-W176.
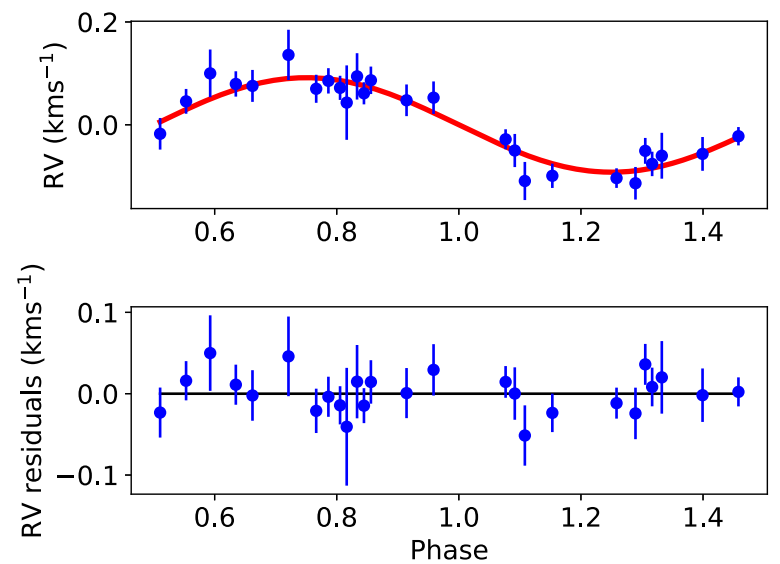

Figure 5. Upper panel: phase-folded CORALIE RV observations of WASP176. Data are shown in blue with the best-fit model derived from the MCMC analysis shown in red. Lower panel: residuals from the RV fit.

(The data used to create this figure are available.)

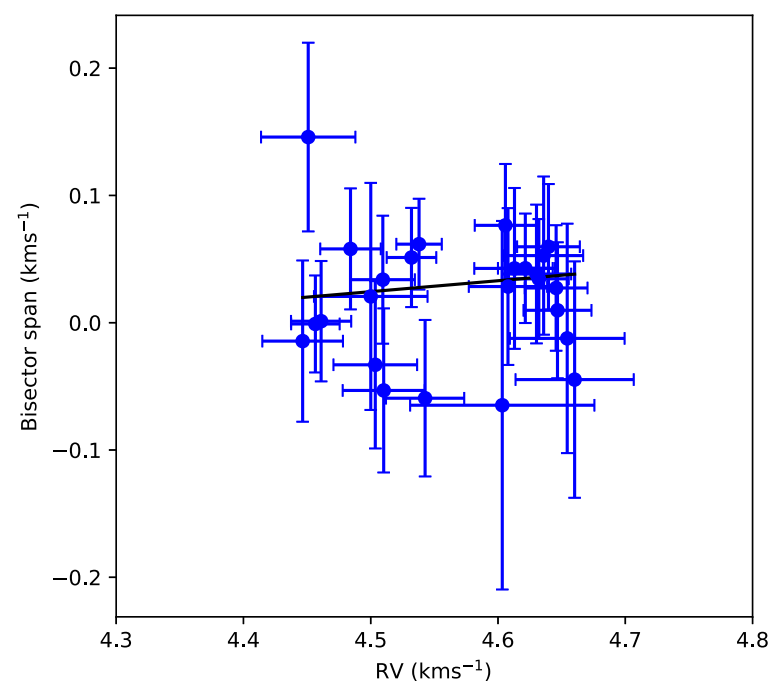

Figure 6. CORALIE RV bisector span as a function of RV for WASP-176. The solid black line shows the best weighted linear fit to the data. The lack of any significant gradient supports the assumption that the RV signal is produced by a planetary companion. The aspect ratio of the two axes is unity.

\subsubsection{IAC80}

A full transit of WASP-150b was observed on 2015 July 7 using the CAmara MEjorada Ligera del Observatorio del Teide $(\text { CAMELOT })^{26}$ camera installed on the IAC $80^{27}$ telescope at the Observatorio del Teide, Tenerife, Spain. CAMELOT contains a $2048 \times 2048$ back-illuminated e2v CCD, providing a field of view of $10 ! 4 \times 10 ! 4$, with a pixel scale of 0 !! 304 .

Data were reduced using standard routines of IRAF (Tody 1986, 1993). All images were bias and flat subtracted and differential photometry was carried out using IDL DAOPHOT-Type Photometry Procedures ${ }^{28}$ (Stetson 1987). Among the several stars appearing on the field of view of the camera, those showing less dispersion were selected to produce

\footnotetext{
$\overline{26}$ http://vivaldi.ll.iac.es/OOCC/iac-managed-telescopes/iac80/camelot/

27 http://www.iac.es/OOCC/instrumentation/iac80/

28 https://idlastro.gsfc.nasa.gov/contents.html
} 
Table 2

Photometric Follow-up of WASP-150

\begin{tabular}{|c|c|c|c|c|c|}
\hline Instrument & Date (Night of) & Filter & No. Points & Average Cadence (s) & Comment \\
\hline NITES & 2014 Sep 11 & None & 805 & 26 & Noisy egress \\
\hline RISE & 2015 May 23 & $V+R$ & 14600 & 1 & No pre-transit OOT \\
\hline IAC80 & 2015 Jul 7 & Johnson-Bessel $B$ & 230 & 75 & Full transit observed \\
\hline TCS & 2015 Jul 24 & Johnson-Cousins $R$ & 13500 & 1 & Full transit observed \\
\hline RISE & 2015 Jul 24 & $V+R$ & 12140 & 1 & Missed egress \\
\hline Cassini & 2015 Aug 27 & Johnson $I$ & 482 & 47 & Full transit observed \\
\hline CAHA & 2015 Aug 27 & Cousins $I$ & 421 & 46 & Full transit observed \\
\hline
\end{tabular}
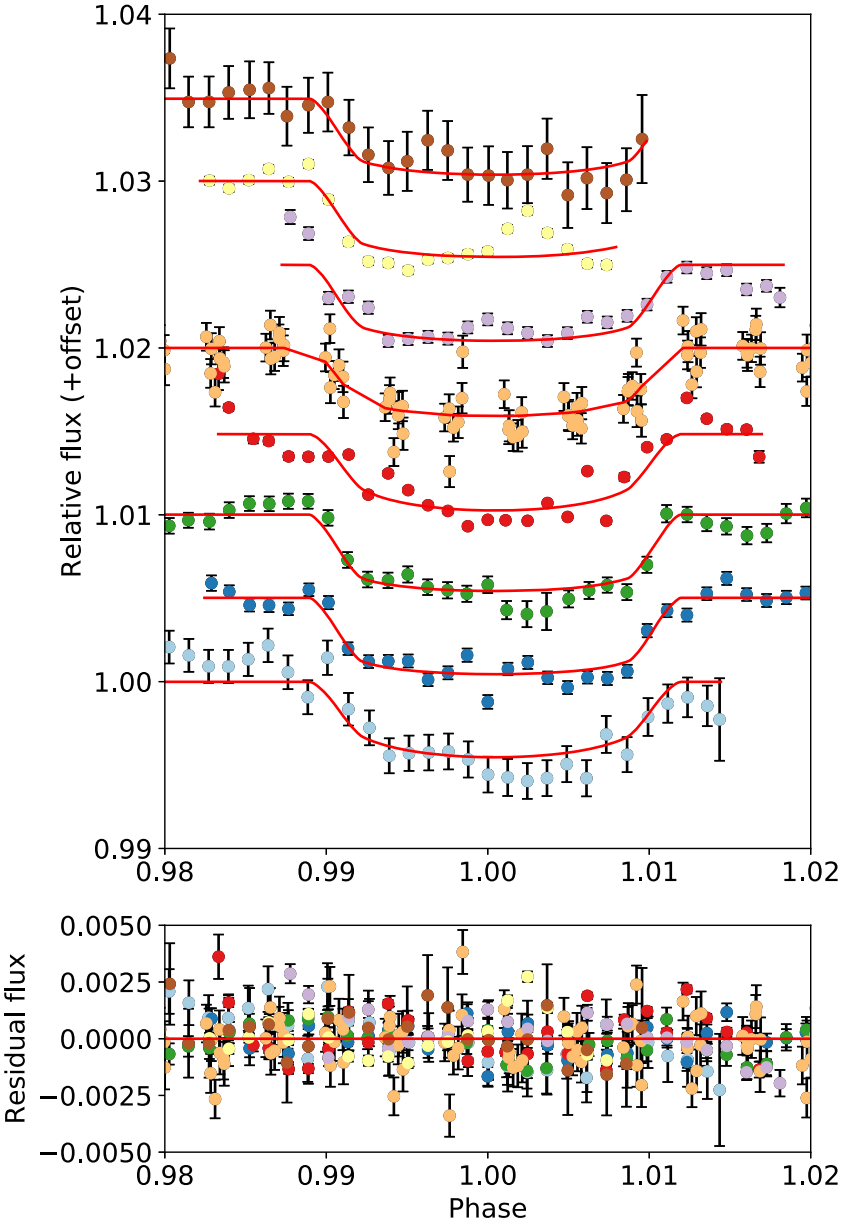

Figure 7. Upper panel: individual WASP-150 light curves binned to 10 minutes (TESS data are left unbinned). From the top down the light curves are from NITES, RISE, RISE, TESS, TCS, Cassini, CAHA, and IAC80. The red curves show the best fit from the MCMC. Lower panel: best-fit residuals colored as in the upper panel.

(The data used to create this figure are available.)

an average reference star and obtain the final light curve. We used a fixed aperture radius of 13 pixels, which minimized the rms scatter in the out-of-transit data.

\subsubsection{CAHA $1.23 \mathrm{~m}$}

The transit on 2015 August 27 was observed with the DLRMKIII camera fed by the Zeiss $1.23 \mathrm{~m}$ Centro Astronómico Hispano-Alemán telescope. During the observations the sky was clear besides a couple of intervals when some thin clouds passed in front of the target. The observations were carried out
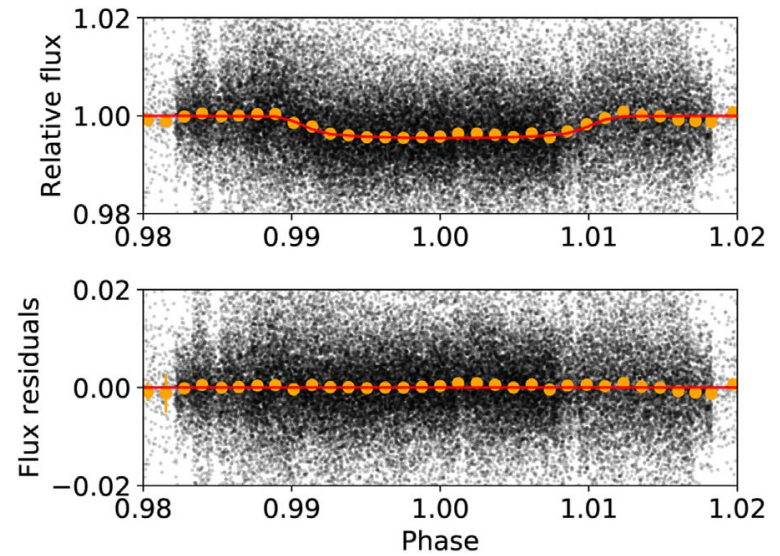

Figure 8. Upper panel: combined photometry data for WASP-150 binned to 10 minutes and including the best fit from the MCMC. Lower panel: the MCMC fit residuals.

using the defocussing technique, which allowed the use of longer exposures compared to in-focus observations, without the risk of saturation (Southworth et al. 2009).

The data reduction was performed using standard methods making use of the DEFOT pipeline (Southworth et al. 2009, 2014). In brief, each scientific image was calibrated using a master bias and a master flat. The fluxes detected from the target and comparison stars were then obtained via aperture photometry, selecting the aperture sizes that minimized the scatter of the light curves. A relative-flux light curve was then obtained for the target star relative to an optimally weighted composite comparison star constructed from the light curves of comparison stars present in the same field of view, to account for atmospheric and instrumental changes occurring during the observations.

\subsubsection{Cassini $1.52 \mathrm{~m}$}

On 2015 August 27 WASP-150b was simultaneously observed with the Cassini $1.52 \mathrm{~m}$ telescope at the Astronomical Observatory of Bologna in Loiano (Italy), thus performing the two-site observational strategy (Ciceri et al. 2013). The Cassini has a focal ratio of $f / 8$, a focal length of $12 \mathrm{~m}$, and is equipped with a back-illuminated CCD with $1300 \times 1340$ pixels and a pixel size of $20 \mu \mathrm{m}$. A focal reducer makes the telescope an $\mathrm{f} /$ 5 , so that its plate scale is 0.58 pixel $^{-1}$ and the field of view is $13^{\prime} \times 12^{\prime}$.6. The autoguided observations were performed with the defocussing technique (to improve the photometric precision), though the level of defocussing was limited in order to avoid blending from a fainter star a few arcsec away. The data were reduced using the DEFOT code, as described in the preceding section. 


\subsubsection{RISE}

Two partial transits of WASP-150b were observed on 2015 May 23 and July 24 with the Rapid Imaging Search for Exoplanets $^{29}$ (RISE) optical camera, installed on the $2 \mathrm{~m}$ robotic Liverpool Telescope (Gibson et al. 2008; Steele et al. 2008) at the Observatorio del Roque de los Muchachos, La Palma, Spain. The camera is a frame transfer e2v CCD of $1024 \times 1024$ pixels, which has a field of view of $9 ! 2 \times 9 ! 2$.

Bias and flat reduced images were provided by the telescope pipeline. The light curves were extracted following the standard procedures described in Section 4.1.1, using a fixed aperture radii of 5.5 and 7 pixels, for the first and second night respectively.

\subsubsection{TCS}

On 2015 July 24, WASP-150b was simultaneously observed with the Telescopio Carlos Sánchez ${ }^{30}$ (TCS), a $1.52 \mathrm{~m}$ telescope installed at the Observatorio del Teide, once again performing the two-site observational strategy (Ciceri et al. 2013). We used the WIDE FASTCAM camera, a $1024 \times 1024$ pixels EMCCD detector, coupled to an optical design (Murga et al. 2014) that is able to provide a field of view of $\sim 8^{\prime} \times 8^{\prime}$. This camera provides small readout times and low electronic noise, which allow us to precisely measure exoplanet transit timings.

All images were bias and flat subtracted, and light curves were extracted using similar procedures to those described in Section 4.1.1. In this case, a fixed radius aperture of 11.5 pixels was selected.

\subsubsection{NITES}

A transit of WASP-150b was obtained on 2014 September 11 using the Near Infra-red Transiting ExoplanetS telescope (NITES; McCormac et al. 2014) on La Palma. The data were reduced in PYTHON using CCDPROC (Craig et al. 2015). Master, dark, and flat biases were created using the standard process. Non-variable nearby comparison stars were selected by hand, and aperture photometry was extracted using SEP (Bertin \& Arnouts 1996; Barbary 2016).

\subsubsection{TESS}

The TESS (Ricker et al. 2015) observed WASP-150b during its northern hemisphere campaign. WASP-150b was observed in the full frame images of sectors 14 and 20. To account for the blending in the TESS light curve (TESS pixels are 21" square) this data was included with a variable dilution term in the MCMC modeling for WASP-150b. This light curve was extracted from the full frame images using the Eleanor extraction pipeline (Feinstein et al. 2019) utilizing the background subtraction and systematics removal packages.

\subsection{WASP- $176 b$}

To gather the necessary follow-up photometry for WASP$176 \mathrm{~b}$, a number of telescopes were used. These are summarized in Table 3 along with observation dates, photometric filters, and transit notes. The following sections detail the follow-up.

\footnotetext{
${ }^{29}$ https://telescope.livjm.ac.uk/TelInst/Inst/RISE/

${ }^{30}$ http://www.iac.es/OOCC/instrumentation/telescopio-carlos-sanchez/
}

The light curves are shown in Figures 9 and 10. Data files can be found at https://github.com/BenCooke95/W150-W176.

\subsubsection{Cassini $1.52 \mathrm{~m}$}

A partial transit of WASP-176b was recorded with the Cassini $1.52 \mathrm{~m}$ telescope on 2018 June 30. Unfortunately, cloud prevented observing the start of the transit. The observations were performed by defocussing the telescope for improving the photometric precision and using autoguiding. The data were reduced as discussed in Section 4.1.3. We detrended the light curve to remove slow instrumental and astrophysical trends, by fitting a straight line to the out-oftransit data.

\subsubsection{NITES}

A total of two transits were obtained using NITES (McCormac et al. 2014) on La Palma. The data were reduced in PYTHON using CCDPROC (Craig et al. 2015). Master, dark, and flat biases were created using the standard process on each night. Twenty-one images of each type were used for the master calibration frames. Non-variable nearby comparison stars were selected by hand, and aperture photometry was extracted using SEP (Bertin \& Arnouts 1996; Barbary 2016). The aperture photometry radii were chosen to minimize the dispersion in the data points out of transit.

\subsubsection{RISE-2}

WASP-176 was observed with RISE-2 mounted on the $2.3 \mathrm{~m}$ telescope situated at Helmos observatory in Greece. RISE-2 has a CCD size of $1 K \times 1 K$ with a pixel scale of 0 " 51 and a field of view of $9^{\prime} \times 9^{\prime}$ (Boumis et al. 2010). The data were reduced using master bias and flat frames, created using the standard process on each night. Non-variable nearby comparison stars were selected by hand and aperture photometry was extracted using SEP (Bertin \& Arnouts 1996; Barbary 2016). The aperture photometry radii were chosen to minimize the dispersion in the data points out of transit.

\subsubsection{TRAPPIST}

TRAPPIST-North (Gillon et al. 2017; Barkaoui et al. 2019) observed one full transit of WASP-176b on 2018 June 26. TRAPPIST-North is a $60 \mathrm{~cm}$ robotic telescope installed in spring 2016 at Oukaimeden Observatory in Morocco. TRAPPIST-North is a northern twin of TRAPPIST-South (Gillon et al. 2011; Jehin et al. 2011). TRAPPIST-North has an $f / 8$ Ritchey-Chretien optical design. It is equipped with a thermoelectrically cooled $2048 \times 2048$ deep-depletion Andor iKon-L CCD camera that has a pixel size of $13.5 \mu \mathrm{m}$, which translates into a $0 . \prime 60$ pixel $^{-1}$ image scale and a field of view of 19 ! $\times 19$ ! 8 . Data reduction consisted of standard calibration steps (bias, dark, and flat-field corrections) and subsequent aperture photometry using IRAF/DAOPHOT (Tody 1986). Extraction of fluxes of selected stars using aperture photometry was performed with IRAF/DAOPHOT (as described in Gillon et al. 2013).

During the TRAPPIST-North observations of WASP-176 the telescope underwent a meridian flip at JD 2458296.6355. To counter this problem the pre- and post-meridian flip data are treated separately. 
Table 3

Photometric Follow-up of WASP-176

\begin{tabular}{|c|c|c|c|c|c|}
\hline Instrument & Date (Night of) & Filter & No. Points & Average Cadence (s) & Comment \\
\hline SPECULOOS-Io & 2018 Jun 14 & Sloan $z^{\prime}$ & 1127 & 21 & Missed egress \\
\hline TRAPPIST-North & 2018 Jun 26 & $I+z$ & 708 & 28 & Full but no out of transit (meridian flip) \\
\hline Cassini & 2018 Jun 30 & Johnson $V$ & 123 & 119 & Missed ingress (cloud) \\
\hline NITES & 2018 Aug 4 & Johnson-Bessel $R$ & 345 & 32 & Missed ingress \\
\hline NITES & 2018 Aug 8 & Johnson-Bessel $I$ & 676 & 32 & Missed egress \\
\hline RISE-2 & 2018 Sep 24 & $V+R$ & 1929 & 11 & Missed ingress \\
\hline
\end{tabular}
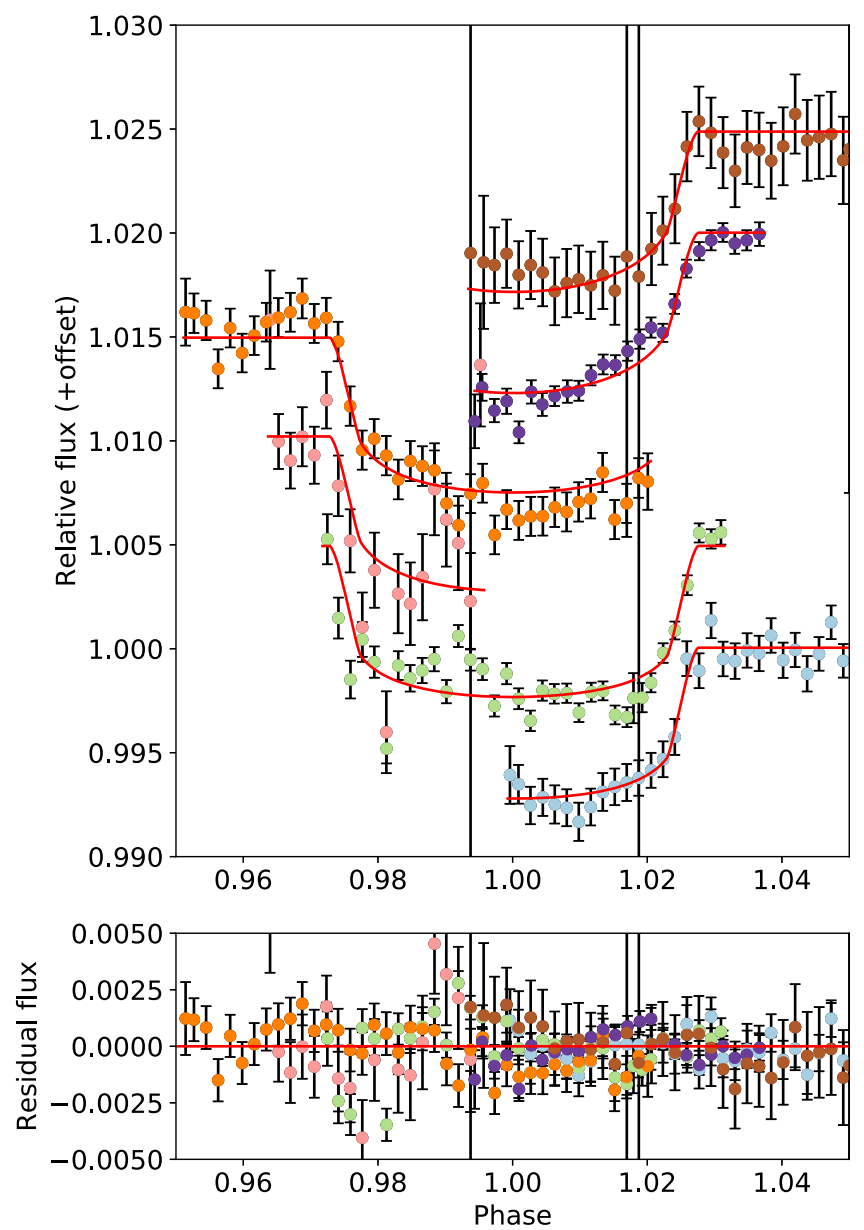

Figure 9. Upper panel: individual WASP-176 light curves binned to 10 minutes. From the top down the light curves are from NITES, TRAPPIST, NITES, SPECULOOS, Cassini, and RISE-2. The red curves show the best fit from the MCMC. Lower panel: best-fit residuals colored as in the upper panel.

(The data used to create this figure are available.)

\subsubsection{SPECULOOS}

One partial transit of WASP-176b was observed with SPECULOOS-Io, one of the four telescopes of the Search for habitable Planets EClipsing ULtra-cOOl Stars (SPECULOOS)South facility (Burdanov et al. 2018; Delrez et al. 2018; Gillon 2018; Jehin et al. 2018), which is located at ESO Paranal Observatory (Chile). Each telescope is a robotic RitcheyChretien $(f / 8)$ telescope of $1 \mathrm{~m}$ diameter. They are equipped with Andor iKon-L Peltier-cooled deeply depleted $2 \mathrm{~K} \times 2 \mathrm{~K}$ $\mathrm{CCD}$ cameras, with good sensitivities in the very near-infrared up to $1 \mu \mathrm{m}$. The field of view of each telescope is $12^{\prime} \times 12^{\prime}$ and the pixel scale is 0 !' 35 pixel $^{-1}$. The calibration and
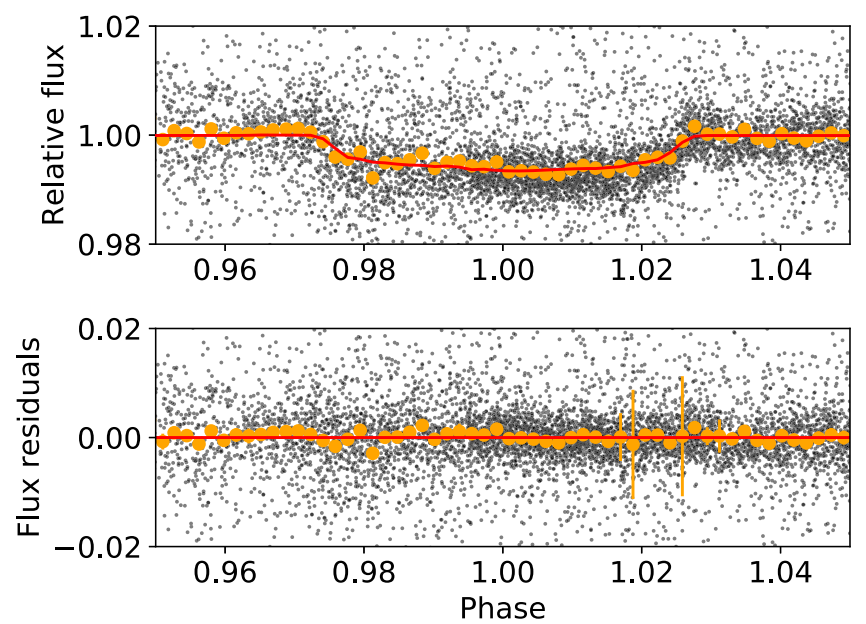

Figure 10. Upper panel: combined photometry data for WASP-176 binned to 10 minutes and including the best fit from the MCMC. Lower panel: MCMC fit residuals.

photometric reduction of the data were performed as described in Gillon et al. (2013).

\section{High Spatial Resolution Follow-up}

WASP-150 was observed on four occasions, 2015 November 15, 2016 March 9 and 10, and 2016 May 6, using the FASTCAM camera (Oscoz et al. 2008) installed on the $1.52 \mathrm{~m}$ Telescopio Carlos Sánchez (TCS) located at the Observatorio del Teide, Tenerife, Spain. FASTCAM is a EMCCD camera with $512 \times 512$ pixels, with a physical pixel size of $16 \mu \mathrm{m}$, which makes a field of view of 21 !" $2 \times 21$ !" 2 . Thanks to the very low noise and fast readout speed of the EMCCD array, this camera is appropriate for Lucky Imaging (LI) observations.

During each of the four observing nights, 10,000 individual frames of WASP-150 were collected in the Johnson-Cousins $I$ filter, except on the 2015 November 15 night, for which a clear filter was used due to the dusty weather conditions (calima). Each individual frame had an exposure time of $50 \mathrm{~ms}$. In total, $500 \mathrm{~s}$ and $1500 \mathrm{~s}$ of data were gathered of WASP-150, with the clear and $I$-band filters, respectively.

Using the FASTCAM dedicated software developed at the Universidad Politécnica de Cartagena (Labadie et al. 2010; Jódar et al. 2013), each individual frame was bias-subtracted, aligned and co-added, and then processed in order to construct a high resolution long-exposure image. For each night's data set, we took a high resolution image constructed by co-addition of the best $30 \%$ of images, thus making a $150 \mathrm{~s}$ total exposure time. No close companion was detected, only a $\Delta m_{I}=2.17 \pm 0.03 \mathrm{mag}$ fainter star at a distance of 10 " $58 \pm 0$ " 05 . Figure 11 shows the contrast light curve that was computed based on the scatter within the annulus as a 


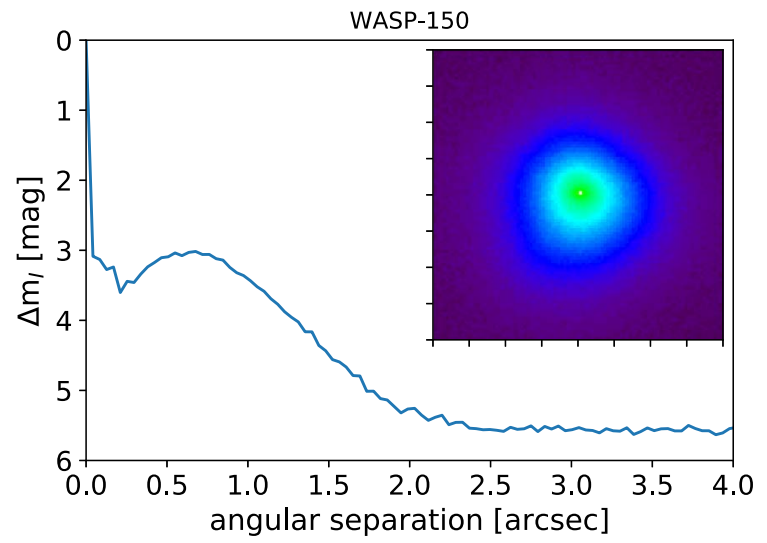

Figure 11. $I$-band magnitude contrast as a function of angular separation up to $4 ! " 0$ of WASP-150. The solid line indicates the $5 \sigma$ detection limit for the primary star. The inset shows the 2 ." $0 \times 2$ !. 0 combined image of WASP- 150 . North is up and east is left.

function of angular separation from the target centroid (see, e.g., Gauza et al. 2015).

\section{Results}

\subsection{WASP-150b}

\subsubsection{Stellar Parameters}

To determine the stellar parameters of WASP-150 a spectral analysis of the SOPHIE spectra was carried out. The results of this analysis are shown in Table 4.

Additionally, the second data release of Gaia (Gaia Collaboration et al. 2016, 2018) provided some of the stellar parameters presented in Table 5. Gaia was also searched for close companions of WASP-150. No significant companion was found.

The open-source stellar modeling code BAGEMASS ${ }^{31}$ (Maxted et al. 2015) was then used to estimate the age and mass of WASP-150. BAGEMASS uses the GARSTEC stellar evolution code (Weiss \& Schlattl 2008) to calculate model grids of individual stars. A Bayesian method then samples the posterior distributions on mass and age. The results of this analysis are presented in Table 6.

The best-fit evolutionary track and isochrone produced from the BAGEMASS analysis are shown in Figure 12 along with the $1 \sigma$ uncertainties. This plot also includes the posterior distribution produced by the EXOFASTv2 analysis (see Section 6.1.2).

Figure 12 includes a small collection of points above the main area of convergence. These data are not affected by increasing the run time of the MCMC analysis or by increasing the burn-in period, thus we do not believe them to be an artifact of unfinished fitting. However, since the significance of this region is $\lesssim 10$ times lower than the peak we do not find it impactful.

\subsubsection{EXOFASTv2 Analysis}

To perform simultaneous fitting of the SuperWASP detection, the SOPHIE RVs, and the follow-up photometry we used the fitting code EXOFASTv2 (Eastman 2017; Eastman et al. 2019). This tool is designed to fit all the available data and ensure consistency between derived stellar and planetary parameters. EXOFASTv2 explores the given parameter space

\footnotetext{
$\overline{31 \text { https://sourceforge.net/projects/bagemass/ }}$
}

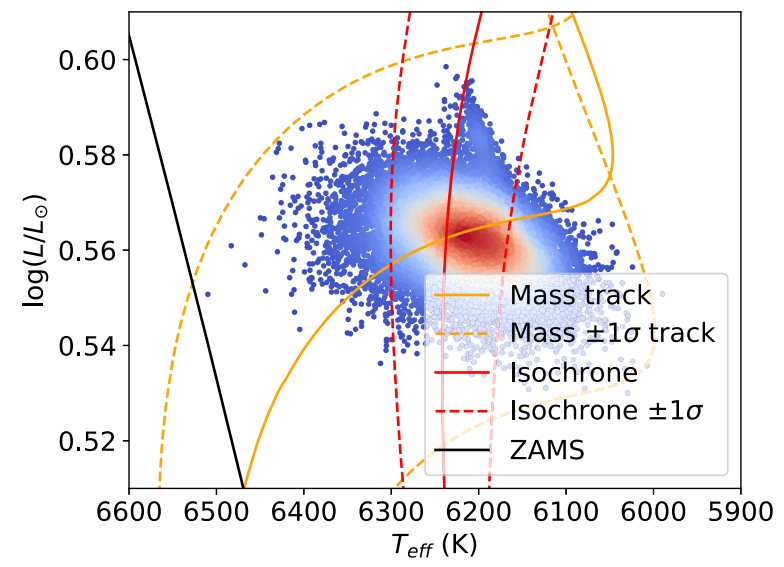

Figure 12. BAGEMASS analysis of WASP-150. The black line shows the zero age main sequence (ZAMS). The orange line shows the best-fit mass track with the dotted lines showing the $\pm 1 \sigma$ mass tracks. The red line shows the isochrone relating to the best-fit age with the dotted lines showing the $\pm 1 \sigma$ isochrones. Finally the density of the EXOFASTv2 samples is shown in the color scale of the plotted posterior distribution.

Table 4

Stellar Parameters from Spectral Analysis

\begin{tabular}{lcc}
\hline \hline Parameter (unit) & WASP-150 & WASP-176 \\
\hline Spectral type & F8 & F9 \\
$T_{\text {eff }}(\mathrm{K})$ & $6250 \pm 80$ & $6100 \pm 100$ \\
$\log g$ & $4.23 \pm 0.13$ & $4.0 \pm 0.2$ \\
{$[\mathrm{Fe} / \mathrm{H}]$} & $0.18 \pm 0.11$ & $0.15 \pm 0.08$ \\
$v \sin i\left(\mathrm{~km} \mathrm{~s}^{-1}\right)$ & $8.82 \pm 0.95^{\mathrm{a}}$ & $3.8 \pm 1.0^{\mathrm{b}}$ \\
\hline
\end{tabular}

Notes.

${ }^{\mathrm{a}}$ Assuming a microturbulance of $0.78 \pm 0.05 \mathrm{~km} \mathrm{~s}^{-1}$ from the Doyle et al. (2013) calibration.

${ }^{\mathrm{b}}$ Assuming a macroturbulance of $5.1 \pm 0.7 \mathrm{~km} \mathrm{~s}^{-1}$ from the Doyle et al. (2013) calibration.

Table 5

Stellar Parameters from Gaia DR2

\begin{tabular}{|c|c|c|}
\hline Parameter (unit) & WASP-150 & WASP-176 \\
\hline Parallax (mas) & $1.865 \pm 0.020$ & $1.731 \pm 0.036$ \\
\hline Distance (pc) & $536 \pm 6$ & $578 \pm 12$ \\
\hline PM R.A. $\left(\operatorname{mas} \mathrm{yr}^{-1}\right)$ & $-4.289 \pm 0.041$ & $-6.192 \pm 0.064$ \\
\hline PM Decl. $\left(\right.$ mas $\left.\mathrm{yr}^{-1}\right)$ & $7.000 \pm 0.040$ & $-4.954 \pm 0.062$ \\
\hline$T_{\text {eff }}(\mathrm{K})$ & $6093_{-70}^{+188}$ & $5902_{-36}^{+20}$ \\
\hline$R_{\star}\left(R_{\odot}\right)$ & $1.706_{-0.101}^{+0.040}$ & $1.945_{-0.013}^{+0.024}$ \\
\hline$L_{\star}\left(L_{\odot}\right)$ & $3.616 \pm 0.0 .073$ & $4.136 \pm 0.130$ \\
\hline
\end{tabular}

Table 6

Stellar Properties from BAGEMASS

\begin{tabular}{lcc}
\hline \hline Parameter (Unit) & WASP-150 & WASP-176 \\
\hline Mass $\left(M_{\odot}\right)$ & $1.346 \pm 0.029$ & $1.270 \pm 0.025$ \\
$\tau_{\text {iso }}(\mathrm{Gyr})$ & $2.950 \pm 0.229$ & $4.810 \pm 0.191$ \\
{$[\mathrm{Fe} / \mathrm{H}]_{\text {init }}$} & $0.204 \pm 0.079$ & $0.215 \pm 0.069$ \\
\hline
\end{tabular}

through a differential evolution Markov chain method using 30,000 steps. We use the Gelman-Rubin statistic (Gelman et al. 2003) to check the mixing of the chains as proposed by Ford (2006). We fit a total of 50 free parameters, these are the parameters presented in Table 7 as well as the limb-darkening 
Table 7

System Parameters from the MCMC Analysis

\begin{tabular}{|c|c|c|}
\hline Parameter (unit) & WASP-150b & WASP-176b \\
\hline$T_{0}(\mathrm{HJD})$ & $7217.2614_{-0.0004}^{+0.0004}$ & $8234.1771_{-0.0007}^{+0.0007}$ \\
\hline$P$ (days) & $5.6442_{-0.0}^{+0.0}$ & $3.8991_{-0.0}^{+0.0}$ \\
\hline$\Delta F$ & $0.0044_{-0.0001}^{+0.0001}$ & $0.0064_{-0.0002}^{+0.0002}$ \\
\hline$T_{14}$ (days) & $0.1299_{-0.0015}^{+0.001}$ & $0.2147_{-0.0019}^{+0.0021}$ \\
\hline$b$ & $0.758_{-0.014}^{+0.011}$ & $0.347_{-0.12}^{+0.098}$ \\
\hline$i\left(^{\circ}\right)$ & $84.01_{-0.2}^{+0.25}$ & $86.7_{-1.1}^{+1.3}$ \\
\hline$e$ & $0.3775_{-0.0029}^{+0.0038}$ & 0.0 (Fixed) \\
\hline$M_{\star}\left(M_{\odot}\right)$ & $1.394_{-0.049}^{+0.07}$ & $1.345_{-0.13}^{+0.08}$ \\
\hline$R_{\star}\left(R_{\odot}\right)$ & $1.651_{-0.03}^{+0.024}$ & $1.925_{-0.044}^{+0.047}$ \\
\hline$\rho_{\star}\left(\rho_{\odot}\right)$ & $0.439_{-0.025}^{+0.032}$ & $0.263_{-0.032}^{+0.03}$ \\
\hline $\log g_{\star}(\mathrm{cgs})$ & $4.147_{-0.02}^{+0.027}$ & $3.995_{-0.053}^{+0.037}$ \\
\hline$T_{\text {eff }}(\mathrm{K})$ & $6218.0_{-45.0}^{+49.0}$ & $5941.0_{-79.0}^{+77.0}$ \\
\hline$[\mathrm{Fe} / \mathrm{H}]$ & $0.156_{-0.089}^{+0.1}$ & $0.164_{-0.082}^{+0.081}$ \\
\hline$M_{\mathrm{pl}}\left(M_{\mathrm{J}}\right)$ & $8.46_{-0.2}^{+0.28}$ & $0.855_{-0.069}^{+0.072}$ \\
\hline$R_{\mathrm{pl}}\left(R_{\mathrm{J}}\right)$ & $1.07_{-0.025}^{+0.024}$ & $1.505_{-0.045}^{+0.05}$ \\
\hline$\rho_{\mathrm{pl}}\left(\rho_{\mathrm{J}}\right)$ & $6.44_{-0.47}^{+0.50}$ & $0.234_{-0.032}^{+0.032}$ \\
\hline $\log g_{\mathrm{pl}}(\mathrm{cgs})$ & $4.263_{-0.023}^{+0.024}$ & $2.972_{-0.053}^{+0.047}$ \\
\hline$T_{\mathrm{pl}}(\mathrm{K})$ & $1460.0_{-11.0}^{+11.0}$ & $1721.0_{-21.0}^{+28.0}$ \\
\hline$a(\mathrm{au})$ & $0.0694_{-0.0008}^{+0.0011}$ & $0.0535_{-0.0019}^{+0.001}$ \\
\hline
\end{tabular}

parameters for each band used and the offset baselines and variances to account for any errors in normalization.

For the EXOFASTv2 input parameters we take period and epoch from the initial SuperWASP discovery photometry. The metallicity and effective temperature are taken from the results of the spectroscopic analysis. Additionally, we use stellar radius and luminosity as well as parallax and distance from Gaia DR2. Finally we impose a prior on the $V$-band extinction from Schlegel et al. (1998) and Schlafly \& Finkbeiner (2011), which are used to model the stellar properties through SED fitting. Within EXOFASTv2 we use the MESA Isochrones and Stellar Tracks (MIST; Choi et al. 2016; Dotter 2016) to model the star. This produces an age of $2.18_{-0.65}^{+0.58} \mathrm{Gyr}$, which is in reasonable agreement with the BAGEMASS age in Table 6 . The best-fit values, along with uncertainties, are presented in Table 7.

\subsection{WASP-176b}

\subsubsection{Stellar Parameters}

Similar to WASP-150, the stellar parameters of WASP176 are calculated via a spectral analysis of the CORALIE spectra. The results of this analysis are shown in Table 4.

Once again, the second data release of Gaia (Gaia Collaboration et al. 2016, 2018) provided some additional stellar parameters presented in Table 5. Gaia was also searched for close companions of WASP-176.

To determine the age of WASP-176 we again ran BAGEMASS and show the results in Table 6.

The best-fit evolutionary track and isochrone produced from the BAGEMASS analysis are shown in Figure 13 along with the $1 \sigma$ uncertainties. This plot also includes the posterior distribution produced by the MCMC analysis (see Section 6.2.2).

Once again we see that Figure 13 includes a few points above the main peak. As before we find that these data are not affected by increasing the run time of the MCMC analysis or

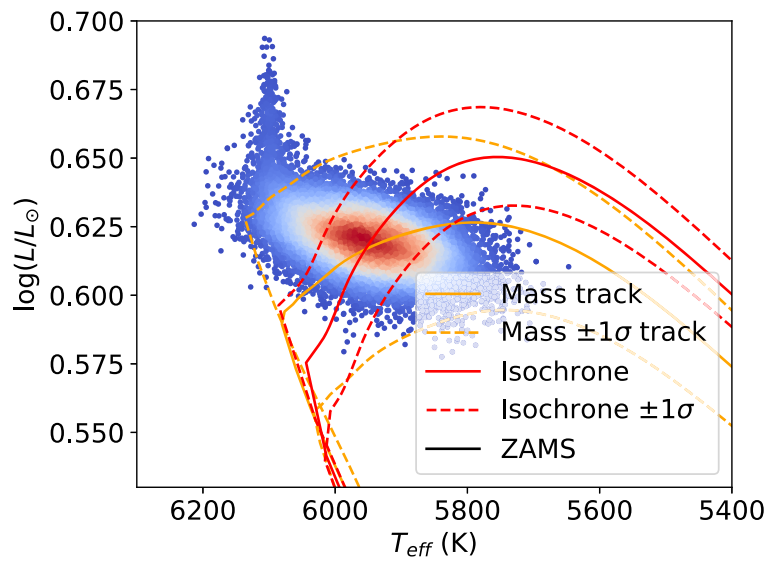

Figure 13. BAGEMASS analysis of WASP-176. The black line shows the zero age main sequence (ZAMS). The orange line shows the best-fit mass track with the dotted lines showing the $\pm 1 \sigma$ mass tracks. The red line shows the isochrone relating to the best-fit age with the dotted lines showing the $\pm 1 \sigma$ isochrones. Finally the density of the MCMC samples is shown in the color scale of the plotted posterior distribution.

by increasing the burn-in period so, again, we do not believe them to be an artifact of unconverged fitting. Since the significance of this region is $\sim 10$ times lower than the peak we do not find it impactful.

\subsubsection{EXOFASTv2 Analysis}

As for WASP-150b we once again use EXOFASTv2 to derive the quoted parameters for WASP-176b. We use the same number of steps, 30,000, and utilize the Gelman-Rubin statistic to check the mixing of the chains. We fit 43 free parameters, these are the parameters presented in Table 7 as well as limb-darkening parameters for each band used and offset baselines and variances to account for any errors in normalization. In the same way as for WASP-150b we take the EXOFASTv2 input parameters from a combination of the WASP discovery photometry (period and epoch), spectroscopic analysis (metallicity and effective temperature), and Gaia DR2 (stellar radius, luminosity, parallax , and distance). Additionally we impose a $V$-band extinction prior from Schlegel et al. (1998) and Schlafly \& Finkbeiner (2011). We use MIST (Choi et al. 2016; Dotter 2016) to model the star. This produces an age of $3.69_{-0.83}^{+1.9} \mathrm{Gyr}$, which is in good agreement with the BAGEMASS age in Table 6 . The best-fit values, along with uncertainties, are presented in Table 7.

The WASP-176b analysis was first conducted allowing for an eccentric orbit. This resulted in $\chi_{\text {ecc }}^{2}=11.423$. Repeating the analysis, this time forcing a circular orbit gives $\chi_{\text {circ }}^{2}=11.358$. Since the discrepancy between these values is negligible it was decided there was insufficient evidence to support eccentricity and thus a circular orbit was assumed.

\section{Discussion and Conclusions}

\subsection{WASP-150b}

WASP-150b is a high-density hot Jupiter on a $5.6442_{-0.0}^{+0.0}$ day orbit around its F8 host. With a mass of of $8.46_{-0.2}^{+0.28} M_{\mathrm{J}}$ and a radius of $1.07_{-0.025}^{+0.024} R_{\mathrm{J}}$, WASP-150b has a density of $6.44_{-0.47}^{+0.50} \rho_{\mathrm{J}}$ placing it among the highest density planets known. Figure 14 shows a plot of all the confirmed exoplanets from the NASA 


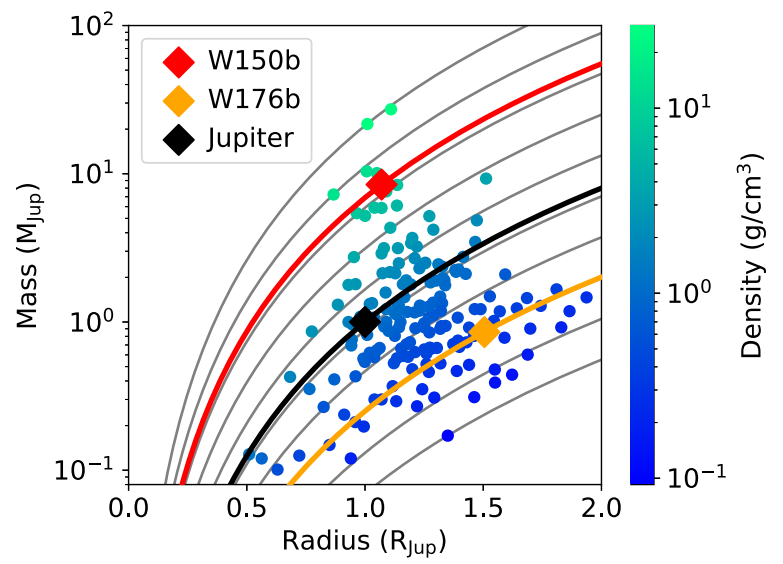

Figure 14. Scatter plot of all confirmed exoplanets from the NASA exoplanet archive with radius and mass known to an accuracy of $\leqslant 10 \%$ and periods of $\leqslant 10$ days. The points are then colored by density in cgs units. WASP-150b is shown as a red diamond and Jupiter is shown as a black diamond for reference (WASP-176b is shown as an orange diamond). The plot also includes lines of constant density to guide the eye.

exoplanet archive with radius and mass known to an accuracy of $\leqslant 10 \%$ and periods of $\leqslant 10$ days. Though large, this density is in line with expectations based on the planets mass (Chabrier et al. 2009).

WASP-150b is also relatively eccentric $\left(e=0.3775_{-0.0029}^{+0.0038}\right)$. Using Equation (3) from Adams \& Laughlin (2006; and assuming $Q_{p} \sim 10^{5}$ ) we see that the approximate circularization timescale for this system is $\sim 5 \mathrm{Gyr}$. Our analysis of this system gives the age of this star as $2.950 \pm 0.229 \mathrm{Gyr}$, well below the circularization timescale, showing that the eccentricity is compatible with the other system parameters. On top of this it has been shown that more massive planets are more inclined to larger eccentricities (Ribas \& Miralda-Escudé 2007) although their origin is still an open question. Additionally, we can use a simple scaling relation to predict the main-sequence lifetime of WASP-150. The following the relation, $t_{\mathrm{MS}}=t_{\mathrm{MS}, \odot}\left(M / M_{\odot}\right)^{-2.5}$, where $t_{\mathrm{MS}, \odot}$ is the main-sequence lifetime of the Sun $(\sim 10 \mathrm{Gyr})$, predicts the main-sequence lifetime of WASP-176 as $\sim 4.3 \mathrm{Gyr}$, much longer than its estimated age.

For some examples of comparable high-mass, hot Jupiters in eccentric orbits see WASP-8b $\left(2.2 M_{\mathrm{J}}, e=0.31\right.$; Queloz et al. 2010), Kepler-75 (9.9 $M_{\mathrm{J}}, e=0.57$; Hébrard et al. 2013), WASP-162b (5.2 $M_{\mathrm{J}}, e=0.43$; Hellier et al. 2018), and HATP-2b (8.74 $M_{\mathrm{J}}, e=0.52$; Bakos et al. 2007). HAT-P-2b was the first exoplanet discovered in this class and has been extensively studied in regards to planet-star interactions. The large mass and eccentricity of WASP-150b, coupled with its high eccentricity, make it an interesting addition to similar studies (Cébron et al. 2013; Salz et al. 2016; de Wit et al. 2017). In particular the short period of this system allows for the collection of entire phase curves, which will enable studies of the evolution of the planetary flux as a function of orbital phase (Lewis et al. 2013). Additionally, the eccentricity means that the planet cannot rotate synchronously. Thus the atmosphere should display time-dependent effects such as atmospheric radiative time constants and tidal luminosities (Laughlin \& Lissauer 2015). Future studies may even be able to detect the manifestation of time-dependent storms (Laughlin et al. 2009).

\subsection{WASP-176b}

WASP-176b is a slightly inflated hot Jupiter orbiting an F9 host star on a 3.8991 $1_{-0.0}^{+0.0}$ day orbit. The MCMC best-fit results presented here give a planetary mass of $0.855_{-0.069}^{+0.072} M_{\mathrm{J}}$ and a planetary radius of $1.505_{-0.045}^{+0.05} R_{\mathrm{J}}$ leading to a density of $0.234_{-0.032}^{+0.032} \rho_{\mathrm{J}}$. Using the same scaling relation as above predicts the main-sequence lifetime of WASP-176 as $\sim 4.77$ Gyr. Using the isochronal age estimated in Section 6.2.1 indicates that the star has evolved past the end of its main-sequence life. This comparison, combined with the stellar surface gravity and density given in Table 7, indicates that the host is a sub-giant. This is further supported by its location on the HR diagram as seen in Figure 13. The planetary radius found here is approximately $25 \%$ larger than expected for a coreless planet, when predicted using the planetary evolution models from Fortney et al. (2007).

WASP-176b is similar to other hot Jupiters (WASP-54b, Faedi et al. 2013; WASP-78b and WASP-79b, Smalley et al. 2012; WASP142b, Hellier et al. 2016; WASP-136b, Lam et al. 2016) in that it receives stronger irradiation from its F-type host than it would from a G-type star, thus leading to inflation. Based on this increased radiation we suggest that the inflation mechanism behind WASP-176b may be due to the deposit of stellar irradiation into the planetary core, consistent with the Class I model presented in Lopez \& Fortney (2016). If so, then this planet may have experienced increasing inflation as its host moved off the main sequence. However this is only a possible solution, additional characterization may give more information to this end.

WASP-176b is a potential target for atmospheric characterization via transmission spectroscopy due to its low density and high equilibrium temperature. If we assume an atmosphere similar in composition to Jupiter $(\mu=2.2 u$, where $u=1.66 \times 10^{-27} \mathrm{~kg}$ is the atomic mass units) then the scale height is found to be $H=k_{B} T / \mu g \sim 500 \mathrm{~km}$ which is smaller, but comparable, to values seen in recent successful atmospheric retrievals (Kirk et al. 2019). However, with a magnitude of only $V=12.01$, the follow-up would be a significant challenge.

We thank the anonymous referee for the helpful comments. B.F. C. acknowledges a departmental scholarship from the University of Warwick. D.P. acknowledges support through a Merit Award from The Royal Society and from the Science and Technology Facilities Council (STFC) ST/P000495/1. M.G. and E.J. are F.R. S.-FNRS Senior Research Associates. K.B. acknowledges support from the Erasmus+ program. L.D. received support from the Gruber Foundation Fellowship. The research leading to these results has received funding from the European Research Council under the FP/2007-2013 ERC grant Agreement No. 336480 and from the ARC grant for Concerted Research Actions financed by the Wallonia-Brussels Federation. D.L.P. acknowledges support from the Royal Society in the form of a Wolfson Merit award and STFC through the Warwick consolidated grant. This work was also partially supported by a grant from the Simons Foundation (PI: Queloz, ID 327127). L.M. acknowledges support from the Italian Minister of Instruction, University and Research (MIUR) through FFABR 2017 fund. L.M. acknowledges support from the University of Rome Tor Vergata through "Mission: Sustainability 2016" fund. The Aristarchos telescope is operated on Helmos 
Observatory by the Institute for Astronomy, Astrophysics, Space Applications and Remote Sensing of the National Observatory of Athens. A.C.C. acknowledges support from the Science and Technology Facilities Council (STFC) consolidated grant No. ST/ R000824/1. D.J.A.B. acknowledges support from the UK Space Agency (UKSA). TRAPPIST-South is a project funded by the Belgian Fonds (National) de la Recherche Scientifique (F.R.S.FNRS) under grant FRFC 2.5.594.09.F. TRAPPIST-North is a project funded by the University of Liège, in collaboration with Cadi Ayyad University in Marrakech (Morocco). G.D. acknowledges support from CONICYT project Basal AFB-170002.

\section{ORCID iDs}

Benjamin F. Cooke (다 https://orcid.org/0000-0002-8824-9956 Don Pollacco (i) https://orcid.org/0000-0001-9850-9697 D. J. A. Brown (1) https://orcid.org/0000-0003-1098-2442 Andrew Collier Cameron (1) https://orcid.org/0000-00028863-7828

Giuseppe D'ago (10 https://orcid.org/0000-0001-9697-7331

C. Hellier (다 https://orcid.org/0000-0002-3439-1439

Alexios Liakos (1) https://orcid.org/0000-0002-0490-1469

Luigi Mancini (i) https://orcid.org/0000-0002-9428-8732

P. F. L. Maxted (1) https://orcid.org/0000-0003-3794-1317

F. J. Pozuelos (1) https://orcid.org/0000-0003-1572-7707

D. Queloz (1) https://orcid.org/0000-0002-3012-0316

Nicole Schanche (1) https://orcid.org/0000-0002-9526-3780

Barry Smalley (10) https://orcid.org/0000-0002-3456-087X

Stéphane Udry (10 https://orcid.org/0000-0001-7576-6236

Pete Wheatley (1) https://orcid.org/0000-0003-1452-2240

\section{References}

Adams, F. C., \& Laughlin, G. 2006, ApJ, 649, 1004

Bakos, G. Á 2018, in Handbook of Exoplanets, ed. H. Deeg \& J. Belmonte (Cham: Springer), 957

Baranne, A., Queloz, D., Mayor, M., et al. 1996, A\&AS, 119, 373

Barbary, K. 2016, JOSS, 1, 58

Barkaoui, K., Burdanov, A., Hellier, C., et al. 2019, AJ, 157, 43

Batalha, N. M. 2014, PNAS, 111, 12647

Bertin, E., \& Arnouts, S. 1996, A\&AS, 117, 393

Boisse, I., Eggenberger, A., Santos, N. C., et al. 2010, A\&A, 523, A88

Borucki, W. J., Koch, D., Basri, G., et al. 2010, Sci, 327, 977

Bouchy, F., Isambert, J., Lovis, C., et al. 2009, EAS Pub. Ser., 37, 247

Boumis, P., Pollacco, D., Steele, I., et al. 2010, in ASP Conf. Ser. 424, 9th Int. Conf. of the Hellenic Astronomical Society, ed. K. Tsinganos, D. Hatzidimitriou, \& T. Matsakos (San Francisco, CA: ASP), 426

Burdanov, A., Delrez, L., Gillon, M., \& Jehin, E. 2018, in Handbook of Exoplanets, ed. H. Deeg \& J. Belmonte (Cham: Springer), 1007

Cébron, D., Le Bars, M., Le Gal, P., et al. 2013, Icar, 226, 1642

Chabrier, G., Baraffe, I., Leconte, J., Gallardo, J., \& Barman, T. 2009, in AIP Conf. Ser. 1094, 15th Cambridge Workshop on Cool Stars, Stellar Systems, and the Sun, ed. E. Stempels (Melville, NY: AIP), 102

Charbonneau, D., Brown, T. M., Latham, D. W., et al. 2000, ApJL, 529, L45

Choi, J., Dotter, A., Conroy, C., et al. 2016, ApJ, 823, 102

Ciceri, S., Mancini, L., Southworth, J., et al. 2013, A\&A, 557, A30

Collier Cameron, A., Bouchy, F., Hébrard, G., et al. 2007a, MNRAS, 375, 951

Collier Cameron, A., Pollacco, D., Street, R. A., et al. 2006, MNRAS, 373, 799

Collier Cameron, A., Wilson, D. M., West, R. G., et al. 2007b, MNRAS, 380,1230

Craig, M. W., Crawford, S. M., Deil, C., et al. 2015, ccdproc: CCD Data Reduction Software, Version 2.1.0, Astrophysics Source Code Library, ascl: 1510.007

Cumming, A., Butler, R. P., Marcy, G. W., et al. 2008, PASP, 120, 531

de Wit, J., Lewis, N. K., Knutson, H. A., et al. 2017, ApJL, 836, L17

Delrez, L., Gillon, M., Queloz, D., et al. 2018, Proc. SPIE, 10700, $107001 \mathrm{I}$

Dotter, A. 2016, ApJS, 222, 8

Doyle, A. P., Smalley, B., Maxted, P. F. L., et al. 2013, MNRAS, 428, 3164

Eastman, J. 2017, EXOFASTv2: Generalized Publication-quality Exoplanet Modeling Code, Version 2, Astrophysics Source Code Library, ascl:1710.003
Eastman, J. D., Rodriguez, J. E., Agol, E., et al. 2019, arXiv:1907.09480 Faedi, F., Pollacco, D., Barros, S. C. C., et al. 2013, A\&A, 551, A73

Feinstein, A. D., Montet, B. T., Foreman-Mackey, D., et al. 2019, PASP, 131, 094502

Ford, E. B. 2006, ApJ, 642, 505

Fortney, J. J., Marley, M. S., \& Barnes, J. W. 2007, AJ, 659, 1661

Gaia Collaboration, Brown, A. G. A., Vallenari, A., et al. 2018, A\&A, 616, A1 Gaia Collaboration, Prusti, T., de Bruijne, J. H. J., et al. 2016, A\&A, 595, A1 Gardner, J. P., Mather, J. C., Clampin, M., et al. 2006, SSRv, 123, 485

Gauza, B., Béjar, V. J. S., Rebolo, R., et al. 2015, MNRAS, 452, 1677

Gelman, A., Carlin, J. B., Stern, H. S., et al. 2003, Bayesian Data Analysis (2nd ed.; London: Chapman and Hall)

Gibson, N. P., Pollacco, D., Simpson, E. K., et al. 2008, A\&A, 492, 603

Gillon, M. 2018, NatAs, 2, 344

Gillon, M., Anderson, D. R., Collier-Cameron, A., et al. 2013, A\&A, 552, A82

Gillon, M., Jehin, E., Magain, P., et al. 2011, EPJWC, 11, 06002

Gillon, M., Triaud, A. H. M. J., Demory, B.-O., et al. 2017, Natur, 542, 456 Grubbs, F. E. 1950, Ann. Math. Statist., 21, 27

Hébrard, G., Almenara, J. M., Santerne, A., et al. 2013, A\&A, 554, A114 Hellier, C., Anderson, D. R., Bouchy, F., et al. 2018, MNRAS, 482, 1379 Hellier, C., Anderson, D. R., Cameron, A. C., et al. 2016, MNRAS, 465, 3693 Henry, G. W., Marcy, G. W., Butler, R. P., et al. 2000, ApJL, 529, L41

Howell, S. B., Sobeck, C., Haas, M., et al. 2014, PASP, 126, 398

Jehin, E., Gillon, M., Queloz, D., et al. 2011, Msngr, 145, 2

Jehin, E., Gillon, M., Queloz, D., et al. 2018, Msngr, 174, 2

Jódar, E., Pérez-Garrido, A., Díaz-Sánchez, A., et al. 2013, MNRAS, 429, 859

Kirk, J., López-Morales, M., Wheatley, P. J., et al. 2019, AJ, 158, 144

Bakos, G. Á, Kovács, G., Torres, G., et al. 2007, ApJ, 670, 826

Kovács, G., Zucker, S., \& Mazeh, T. 2002, A\&A, 391, 369

Labadie, L., Rebolo, R., Femenía, B., et al. 2010, Proc. SPIE, 7735, 77350X

Lam, K. W. F., Faedi, F., Brown, D. J. A., et al. 2016, A\&A, 599, A3

Laughlin, G., Deming, D., Langton, J., et al. 2009, Natur, 457, 562

Laughlin, G., \& Lissauer, J. J. 2015, arXiv:1501.05685

Lewis, N., Showman, A. P., Fortney, J. J., Knutson, H., \& Marley, M. S. 2013, AAS Meeting, 222, 302.02

Lopez, E. D., \& Fortney, J. J. 2016, ApJ, 818, 4

Maxted, P. F. L., Serenelli, A. M., \& Southworth, J. 2015, A\&A, 575, A36

Mayor, M., Marmier, M., Lovis, C., et al. 2011, arXiv:1109.2497

McCormac, J., Skillen, I., Pollacco, D., et al. 2014, MNRAS, 438, 3383

Murga, G., Oscoz, A., López, R., et al. 2014, Proc. SPIE, 9147, 91476Q

Oscoz, A., Rebolo, R., López, R., et al. 2008, Proc. SPIE, 7014, 701447

Pascale, E., Bezawada, N., Barstow, J., et al. 2018, Proc. SPIE, 10698, $106980 \mathrm{H}$

Pepe, F., Bouchy, F., Mayor, M., et al. 2017, in Handbook of Exoplanets, ed. H. J. Deeg \& J. A. Belmonte (Cham: Springer), 190

Pepe, F., Mayor, M., Galland, F., et al. 2002, A\&A, 388, 632

Pepper, J., Pogge, R. W., DePoy, D. L., et al. 2007, PASP, 119, 923

Perruchot, S., Kohler, D., Bouchy, F., et al. 2008, Proc. SPIE, 7014, 70140J

Pollacco, D. L., Skillen, I., Collier Cameron, A., et al. 2006, PASP, 118, 1407

Queloz, D., Anderson, D. R., Collier Cameron, A., et al. 2010, A\&A, 517, L1

Queloz, D., Henry, G. W., Sivan, J. P., et al. 2001, A\&A, 379, 279

Queloz, D., Mayor, M., Weber, L., et al. 2000, A\&A, 354, 99

Rauer, H., Aerts, C., Cabrera, J. \& PLATO Team 2016, AN, 337, 961

Ribas, I., \& Miralda-Escudé, J. 2007, A\&A, 464, 779

Ricker, G. R., Winn, J. N., Vanderspek, R., et al. 2015, JATIS, 1, 014003

Salz, M., Schneider, P. C., Czesla, S., \& Schmitt, J. H. M. M. 2016, A\&A, $585, \mathrm{~L} 2$

Schanche, N., Collier Cameron, A., Hébrard, G., et al. 2019, MNRAS, 483, 5534

Schlafly, E. F., \& Finkbeiner, D. P. 2011, ApJ, 737, 103

Schlegel, D. J., Finkbeiner, D. P., \& Davis, M. 1998, ApJ, 500, 525

Smalley, B., Anderson, D. R., Collier-Cameron, A., et al. 2012, A\&A, 547, A61

Southworth, J., Hinse, T. C., Burgdorf, M., et al. 2014, MNRAS, 444, 776

Southworth, J., Hinse, T. C., Jørgensen, U. G., et al. 2009, MNRAS, 396, 1023

Steele, I. A., Bates, S. D., Gibson, N., et al. 2008, Proc. SPIE, 7014, 70146J

Stetson, P. B. 1987, PASP, 99, 191

Tamuz, O., Mazeh, T., \& Zucker, S. 2005, MNRAS, 356, 1466

Tody, D. 1986, Proc. SPIE, 627, 733

Tody, D. 1993, in ASP Conf. Ser. 52, Astronomical Data Analysis Software and Systems II, ed. R. J. Hanisch, R. J. V. Brissenden, \& J. Barnes (San Francisco, CA: ASP), 173

Weiss, A., \& Schlattl, H. 2008, Ap\&SS, 316, 99

Wheatley, P. J., West, R. G., Goad, M. R., et al. 2018, MNRAS, 475, 4476

Winn, J. N. 2010, in Exoplanets, ed. S. Seager (Tucson, AZ: Univ. Arizona Press), 55 